DER FINDLING

KASPAR HAUSER IN DER LITERATUR 


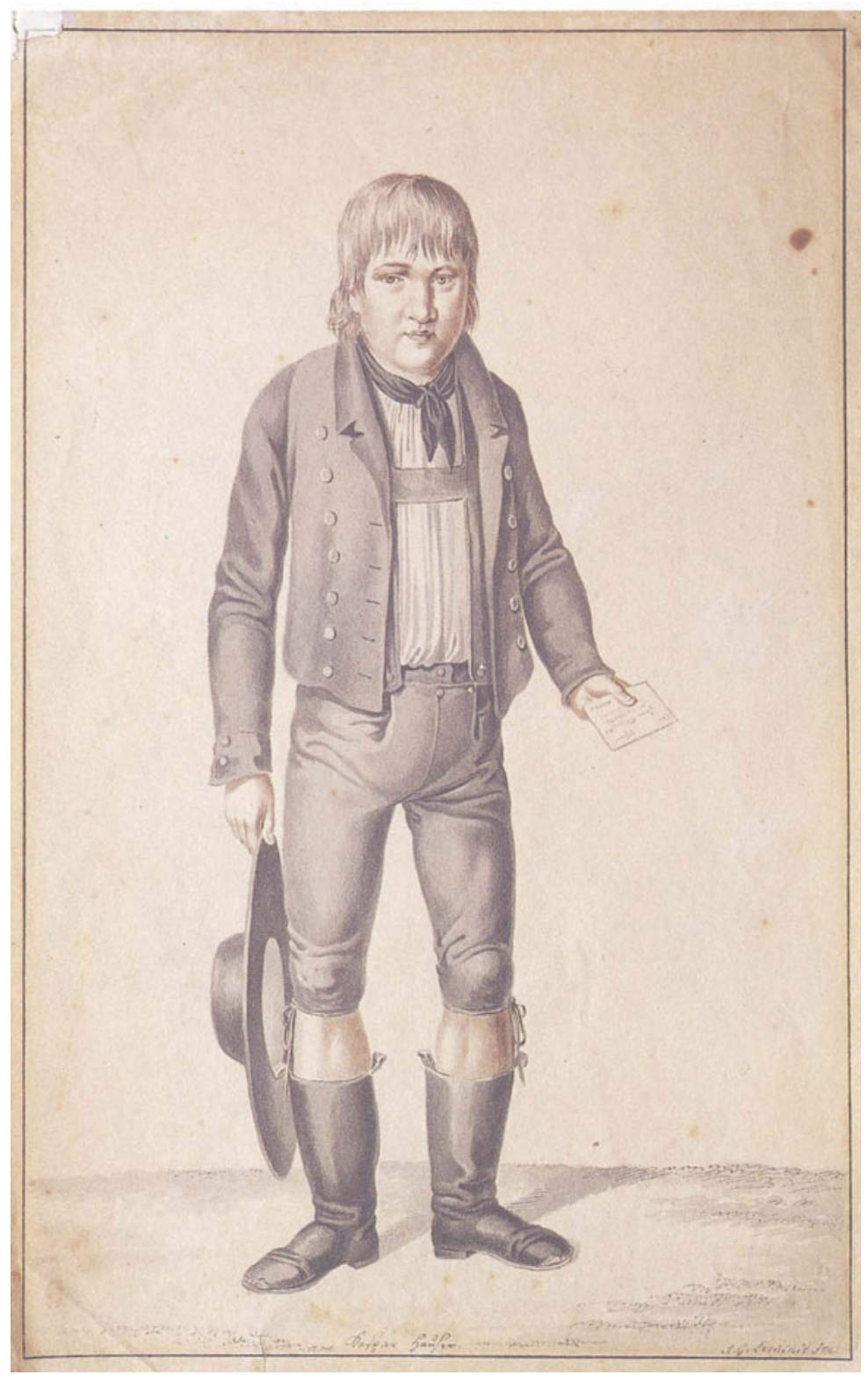

Kaspar Hauser mit dem »Rittmeisterbrief «.

Getuschte Federzeichnung (1828) von Johann Georg Laminit (1775-1848). 


\section{DER FINDLING \\ KASPAR HAUSER IN DER LITERATUR}

Herausgegeben und eingeleitet von Ulrich Struve 
Die Deutsche Bibliothek - CIP-Einheitsaufnahme

Der Findling: Kaspar Hauser in der Literatur / hrsg. und eingeleitet von Ulrich Struve. - Stuttgart, Metzler 1992

NE: Struve, Ulrich [Hrsg.]

ISBN 978-3-476-00786-5

ISBN 978-3-476-03383-3(eBook)

DOI 10.1007/978-3-476-03383-3

Dieses Werk einschließlich aller seiner Teile ist urheberrechtlich geschützt. Jede Verwertung außerhalb der engen Grenzen des Urheberrechtsgesetzes ist ohne Zustimmung des Verlages unzulässig und strafbar. Das gilt insbesondere für Vervielfältigungen, Übersetzungen, Mikroverfilmungen und die Einspeicherung und Verarbeitung in elektronischen Systemen.

(C) 1992 Springer-Verlag GmbH Deutschland Ursprünglich erschienen bei J.B. Metzlersche Verlagsbuchhandlung und Carl Ernst Poeschel Verlag GmbH in Stuttgart 1992 


\section{INHALT}

EINLEITUNG . . . . . . . . . . . . . . . . . . . . 1

Kaspar Hauser : Autobiographie, Den schönsten Tag begrüß ich heut, Albumblatt (1828/29) . . . . . . . . . . . . . 11

JOHANN JACOB LEWERER : Lied (1828) $\ldots \ldots \ldots$

A. C. A. Eschenmayer: Ein Wort über Kaspar Hauser (1832) . 26

Anonym: Das stille Pfarrhaus. (Eine wahre Geschichte.)

(1833) . . . . . . . . . . . . . . . . 29

P.C. Geissler: Caspar Hauser's Ermordung (1833/34) . . . . . 32

M. v. MeILler: Blume, gelegt auf Caspar Hauser's Grab (1834) ........................ 33

J. v.EICHENDORfF : Brief an Theodor v. Schön (1834) . . . . . 35

J. Pirazzi : Über Kaspar Hauser (1834) . . . . . . . . . . . 38

FRITZ GüLl: Caspar Hauser (1834) . . . . . . . . . . . . . . 41

Ludwig Georg Friedrich Seybold : Kaspar Hauser oder der Findling (1834) . . . . . . . . . . . . . . . . 43

Georg Carl Ludwig Schoepfer: Kaspar Hauser, oder: die eingemauerte Nonne (1834) . . . . . . . . . . . 52

Philipp Konrad Marheineke: Das Leben im Leichentuch (1834) . . . . . . . . . . . . . . . . 59

Anonym : Bänkellied (1834) f . . . . . . . . . . 63

Philipp Heinrich Welcker: Tönende Bilder. Kaspar Hauser (1835) . . . . . . . . . . . . . . 65

Anicet Bourgeois / Adolphe Philippe, genannt Dennery:

Gaspard Hauser, Drame en quatre actes (1838) . . . . . . . 73 


\section{INHALT}

»Caspar Hauser« in Peale's New York Museum (1843) . . . . . 74

Vie et aventures de Gaspard Hauser l'idiot de Nuremberg (1860) ...................... . . 76

Karl Gutzkow : Die Söhne Pestalozzi's (1870) . . . . . . . . 77

Georg Friedrich DAumer: Zu Ende geht mein Erdenlauf (1873), Kaspar Hausers Klage (zw. 1858-1875) . . . . . . . 88

Paul Verlaine: Gaspard Hauser chante (1873/81) . . . . . . 91 Übertragungen:

OtTo Haendler: Kaspar Hauser spricht (1903) . . . . . . . 92 Stefan George : Kaspar Hauser singt (1905) . . . . . . . . 92

WOlf BiermanN : Kaspar Hauser singt (1986) . . . . . . . . 93

Wilhelm Herchenbach : Der Findling von Nürnberg (1884) . . . . . . . . . . . . . . . . . . . . . 94

Ludwig BERNDT : Kaspar Hauser der Findling von Nürnberg. Großer Volks-Roman (1896ff.) . . . . . . . . . . . . . . . 106

Kurt Martens : Kaspar Hauser. Drama in vier Akten (1903) . . . . . . . . . . . . . . . . . . . . . 112

Rainer Maria Rilke: Der Knabe (1906) . . . . . . . . . . . 117

Sophie Hoechstetter : Kaspar Hauser (1906), Johannistag in Ansbach (1907), Ein Vorspiel (1919) . . . . . . . . . . . 118

Jakoв Wassermann: Caspar Hauser oder die Trägheit des Herzens $(1907 / 08)$. . . . . . . . . . . . . . . . . . . . . 126

Rudolf Steiner : ̈̈ußerungen zu Kaspar Hauser (1908-1925) . . . . . . . . . . . . . . . . . . . 144

Victor Curt Bihacht : Kaspar Hauser. Ein Spiel (1911) .. . 152

GEORg TRAKL: Kaspar Hauser Lied (1913) . . . . . . . . . . 160

Kurt Matull: Kaspar Hauser - Stummfilm (1915) . . . . . . 162 
Emil Pirchan: Ansbach. Ein Dramolett (1917) . . . . . . . 163

KuRT Tucholsky : Namensänderung (1918) . . . . . . . . . 169

Hans Arp: kaspar ist tot (1919 / 1920 / 1948), Kaspar (1930) . . . . . . . . . . . . . . 170

Kaspar Hauser: Die entartete Prinzeß (1922) . . . . . . . . 174

Klabund: Der arme Kaspar (1922) . . . . . . . . . . . . . 184

Hugo von Hofmannsthal: Der Turm. Ein Trauerspiel $(1923-25 / 1927) \ldots \ldots . \ldots . \ldots 185$

Klara Hofer: Das Schicksal einer Seele (1924) . . . . . . 190

Klaus Mann : Kaspar-Hauser-Legenden (1925) . . . . . . . . 199

Helene Hirschmann: Kaspar Hauser (1925), Kaspar Hausers erstes Weihnachtsfest (1926) _ . . . . . . . . . . . . 208

Thomas Theodor Heine : Jubiläen im Jahre 1926 (1926) Karikatur . . . . . . . . . . . . . . 216

Bernhard Trinius: Kaspar Hauser (1926) . . . . . . . . . . 217

ERICH Ebermayer: Kaspar Hauser. Dramatische Legende

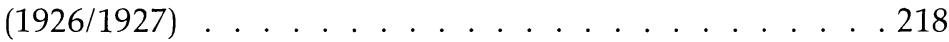

Hans Lindenmeyer : Kaspar Hauser. Historische Ballade im Moritatenstil (1927) . . . . . . . . . . . . . . . . 226

Maria Mathi : Kaspar Hauser (1927) . . . . . . . . . . . . . 229

Herbert Lewandowski: Das Tagebuch Kaspar Hausers (1928) . . . . . . . . . . . . . . . 230

Alfred Kittner : Kaspar-Hauser-Lied (1928) . . . . . . . . . 238

Paul Schröter: Stenographieübungen: Feuerbachs Kaspar Hauser (um 1930) . . . . . . . . . . . . . . . . . 240

JoHANNEs BobRowski: Kaspar Hauser (zw. 1935-1944) . . . . 241 
INHALT

Meret Oppenheim : Kaspar Hauser oder Die Goldene Freiheit

(1942), Ohne mich ohnehin ohne Weg . . . . . . . . . . . . 242

Walter Höllerer : Gaspard (1955) . . . . . . . . . . . . . 247

The Mystery of Caspar Hauser (1956) $\ldots \ldots$. . . . . . . . 250

Peter Härtling : kasper (1958), nachricht von kasper (1961), Kaspar Hauser (1987) ． . . . . . . . . . . . . . . . . . . 251

Hans Holgard : Der Mörder des Kaspar Hauser.

Der Roman eines Schurken (1958/1963) . . . . . . . . . . 254

Dr. Wilhelm KestraneK : Der Fall Kaspar Hauser.

Eine astrologische Untersuchung (1959) . . . . . . . . . 263

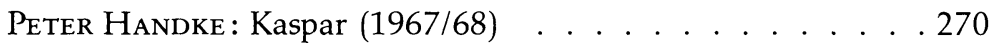

ReINHARd Mey : Kaspar (1968) . . . . . . . . . . . . . 273

Guntram Vesper: Kinder (1970), Ein blinder Passagier steigt zu (1979), Licht in das Leben (1982) . . . . . . . . . 275

Deborah Eibel: Kaspar Hauser in Nuremburg /

Kaspar Hauser in Nürnberg (1972) . . . . . . . . . . . . 287

Werner Herzog : Jeder für sich und Gott gegen alle (1974) . 290

Joachim Minnemann : Kaspar (1976) . . . . . . . . . . 303

Emma Krell-Werth : Gedichte zu »Kaspar Hauser« (1976) . 304

Marcia Southwick : Kaspar Hauser. engl. / dt. (1977) . . . . 307

ERICH FRIED : Klage über die Doppeldeutung (1977) . . . . . . 309

Dieter Forte: Kaspar Hausers Tod (1979) . . . . . . . . . . 311

Robin Fulton: The Story of Kaspar Hauser / Die Geschichte von Kaspar Hauser (1983) . . . . . . . . . . . . . . . . 312 
Swami Alok Chinmayo: Kaspar Hauser 1984. Fragmente.

(1984), I'll be your mirror, reflect what you are . . .

$(1984 / 88) \ldots \ldots \ldots$. . . . . . . . . . . . . . . . . . . .

Thomas LehNeR: Der rätselhafte Kaspar Hauser oder

Schwierigkeiten beim Entziffern der Welt. Hörspiel (1984) .318

Bill Sewell: Kaspar Hauser. engl. / dt. (1985) . . . . . . . . 323

Jürg Amann: Ach, diese Wege sind sehr dunkel (1985) . . . . 325

ENSEMble PRISMA : kaspar hauser-versuch (1985) . . . . . . 333

SuzAnne Vega: Wooden Horse (Caspar Hauser's Song) /

Holzpferdchen (Kaspar Hausers Lied) (1987) . . . . . . . . 335

Katja Lange-Müller : Kasper Mauser - Die Feigheit vorm

Freund (1988) . . . . . . . . . . . . . . . . . . . . . 337

Sibylle Gielen : Ein Mensch wie Kaspar H. Performance (1990) . . . . . . . . . . . . . . . . 344

Danksagung . . . . . . . . . . . . . . . . . . 345

Auswahlbibliographie . . . . . . . . . . . . . . . . . 347

Anmerkungen . . . . . . . . . . . . . . . . 355

Abbildungsverzeichnis, Quellenverzeichnis . . . . . . . . 358 
für Katharina

$\sigma \alpha \sigma$ 